TITLE:

\title{
Improved models of the piezomagnetic field for the 2011 Mw 9.0 Tohoku-oki earthquake
}

\author{
$\operatorname{AUTHOR}(S):$
}

Yamazaki, Ken'ichi

\section{CITATION:}

Yamazaki, Ken'ichi. Improved models of the piezomagnetic field for the 2011 Mw 9.0 Tohoku-oki earthquake. Earth and Planetary Science Letters 2013, 363: 9-15

ISSUE DATE:

2013-02-01

URL:

http://hdl.handle.net/2433/169695

\section{RIGHT:}

(c) 2012 Elsevier B.V.; この論文は出版社版でありません。引用の際には 出版社版をご確認ご利用ください。; This is not the published version. Please cite only the published version. 
1 Improved models of the piezomagnetic field for the $2011 \mathrm{Mw} 9.0$

2 Tohoku-oki earthquake

3

$4 \quad$ Ken'ichi Yamazaki*

5 Miyazaki Observatory, Research Center for Earthquake Prediction, Disaster Prevention

6 Research Institute, Kyoto University, 3884 Kaeda, Miyazaki 889-2161, Japan

7

$8 \quad$ *Corresponding author. Tel.: +81 985651161, Fax: +81 985554005

$9 \quad$ E-mail address: kenichi@rcep.dpri.kyoto-u.ac.jp (K. Yamazaki)

10 


\section{ABSTRACT}

14 To assess the feasibility of observing changes in the magnetic field produced by the

15 piezomagnetic effect, an improved model of the piezomagnetic field corresponding to

16 the Mw 9.0 Tohoku-oki earthquake is presented. In contrast to an earlier study, the

17 proposed model explicitly considers the spatial distribution of slip on the seismic fault,

18 and the results from this new model differ significantly from those of the previous

19 model where slip distributions were ignored. Quantitative aspects of the piezomagnetic

20 effect are discussed through comparisons of data and models. One feature clarified is

that, because the fault rupture is so far offshore, the expected amplitudes are quite small at onshore existing observation sites; consequently, there would have been little chance

23 of observing sizable piezomagnetic signals at inland sites during the Tohoku-oki

24 earthquake. Nevertheless, piezomagnetic signals were reportedly detected at a few sites, possibly indicating that the stress sensitivity or the initial magnetization was larger (by several factors) than assumed. On the other hand, relatively large variations in the magnetic field of up to $10 \mathrm{nT}$ may have occurred offshore. This means that if ocean-bottom sensors had been installed, larger piezomagnetic signals would have been 
30 slip distribution, suggesting that observations of the magnetic field at ocean-bottom

31 sites might provide important constraints on determination of slip models.

32

33 Keywords: piezomagnetic effect; stress sensitivity; 2011 Tohoku-oki earthquake; slip

34 distribution 


\section{Introduction}

mechanical stress, predicts changes in the Earth's magnetic field following a major

constitutive law of the relation between stress changes and magnetization changes has

been proposed, as follows:

where $\Delta M_{i}$ is the change in remanent and induced magnetization, $\Delta T_{i j}$ is the deviatoric

stress tensor, $M_{j}$ is the initial total magnetization (i.e. the sum of induced and remanent

magnetization without changes in stress), and $\beta$ is a proportional coefficient that is

usually referred to as the (piezomagnetic) stress sensitivity. Because of the

piezomagnetic effect, it should be possible to monitor changes in stress in the Earth's 
changes in magnetization in terms of the magnetic Coulomb's law, and further inverted

55

56

57

to changes in stress in terms of eq. (1).

However, the usefulness of the piezomagnetic effect as a tool for monitoring changes in stress is still not clear. Whether or not the piezomagnetic field can be observed depends on the spatial distribution of the piezomagnetic field which, in turn, depends on source type, depth and distance. If the piezomagnetic field has a detectable magnitude that is restricted to just a narrow area, then its detection will be difficult with poorly or sparsely spaced arrays of instruments. Numerical examinations of realistic source models need to be performed in order to assess the detectability of the piezomagnetic field.

The piezomagnetic stress sensitivity is another uncertain factor that determines whether or not the piezomagnetic field is detectable. While the proportional relation (i.e. eq. 1) is partially inferred from considerations based on thermodynamics (e.g. Nakamura and Nagahama, 1997), and the values of stress sensitivity can be determined by theoretical considerations (Stacey and Johnston, 1972), the actual magnetization fraction and type varies from rock to rock and representative values for a particular region must be determined from magnetic anomaly maps, geology and laboratory experiments. Laboratory experiments (e.g. Nagata and Kinoshita, 1967) suggest that 
72 stress sensitivities are on the order of $10^{-9} \mathrm{~Pa}^{-1}$. A stress sensitivity of this order is

usually assumed when the piezomagnetic effect is considered in studies of volcanoes

(e.g. Currenti et al., 2005) and earthquakes (e.g. Okubo et al., 2011). However, these

values are sometimes too small to explain the observed offsets in the magnetic field associated with stress changes (e.g. Nishida et al., 2004; Oshiman et al., 1990; Zhan, 1989). The effective values of the stress sensitivity on the geophysical scale (i.e. larger than the laboratory scale) should be evaluated by comparing observational and theoretical models.

The 2011 Mw 9.0 Tohoku-chihou Taiheiyou-oki earthquake (herein referred to as the Tohoku-oki earthquake), which occurred on the boundary between the Pacific and Eurasian plates, is one event for which the magnitudes of the piezomagnetic field can be examined. The Tohoku-oki earthquake is the largest seismic event to have been observed with a dense network of modern geophysical instruments. Along with seismological and geodetic data, geomagnetic data were obtained for this extreme event. Utada et al. (2011) presented a prompt and comprehensive report on observed variations in the geomagnetic field associated with the Tohoku-oki earthquake. Together with several types of geomagnetic variations that followed the earthquake, they also reported that magnetic field offsets, which probably arose from the piezomagnetic effect, are 
90 actually observed, but they are only up to $1.0 \mathrm{nT}$ at the observation sites. In their

91 conclusions, Utada et al. (2011) presented a negative view on the detectability of the

92 piezomagnetic field.

93 Although the observations reported in Utada et al. (2011) provide constraints on the

94 phenomena that actually occurred at the time of the earthquake, their conclusions about

95 the piezomagnetic effect need to be reconsidered because they are based on

96 oversimplified source models that ignore the spatial distribution of slip on the fault. Any

97 reconsideration should incorporate improved piezomagnetic field models in the hope of

98 clarifying the quantitative nature of the piezomagnetic field and evaluating the

99 usefulness of observing it.

100 The aims of this study are to: (1) provide constraints on the piezomagnetic stress

101 sensitivity around the Tohoku region, near the seismic fault of the Mw 9.0 Tohoku-oki

102 earthquake; and (2) assess the usefulness of the magnetic observations as a tool for

103 detecting stress changes. To these ends, improved models of the piezomagnetic field are

104 presented, and the various models are compared and assessed using the data presented

by Utada et al. (2011). 


\section{Procedures for modeling the piezomagnetic field}

This study presents a new model of the piezomagnetic field in which the spatial of the piezomagnetic field in relation to earthquakes (e.g. Nishida et al., 2007), uniform slip models are employed. However, the slip on a fault is generally heterogeneous, and

113 it is therefore preferable to explicitly consider the spatial distribution of slip. In a

114 uniform slip model, the fault parameters are averaged to obtain a single slip parameter.

115 Such a simplification is valid if the spatial scale of the slip on the fault is smaller than

116 the distance between the observation site and the fault. This criterion is not satisfied in

117 the case of the Tohoku-oki earthquake, for which the spatial scale of the slip and the

118 distance from the surface expression of fault rupture to observational sites are of the

119 order of $100 \mathrm{~km}$. Therefore, the assumption of uniform fault slip used previously by

120 Utada et al. (2011) is likely inadequate in the calculations of the piezomagnetic fields

121 produced by the earthquake. et al. (2011) (herein, referred to as the USGS and UCSB models, respectively), which are derived from seismic wave inversion. Averaged slips from these models were used 
125 in the calculations of Utada et al. (2011). These models were published immediately

126 following the Tohoku-oki earthquake and we can expect further refinements as more

127 complete geophysical data sets are taken in account (e.g. Koketsu et al., 2011).

128 Nevertheless, important characteristics of the Tohoku-oki fault slip are apparent in both

129 the USGS and UCSB models. For example, both models indicate large slip near the

130 trench axis. With these slip distributions, it is possible to evaluate the importance of

131 heterogeneous slip distributions and to compare our results with those of Utada et al.

132 (2011).

133 Piezomagnetic fields that correspond to a heterogeneous slip model are calculated

134 according to the following procedure. The fault plane (i.e. plate boundary) is divided

135 into sub-faults, and on each sub-fault the slip is assumed to be uniform. The total

136 piezomagnetic signal at any point is the sum of the contributions from all sub-faults, and

137 each contribution can be calculated by analytical formulae (Utsugi et al., 2000). Using

138 this procedure, we can calculate the piezomagnetic signals that correspond to

139 heterogeneous slip models for the Tohoku-oki earthquake.

140 Because the above procedure involves formulae derived by Utsugi et al. (2000), all

141 the assumptions involved in the formulations of Utsugi et al. (2000) are also used in the present models. The initial total magnetization of the crust is assumed to be uniform 
143 between the ground surface and a constant Curie point depth. The Earth's crust is

144 approximated by elastic half-space, surface of which locates at sea level. These assumptions are not satisfied in reality, thus producing some uncertainty in the models obtained for the piezomagnetic field. Errors should also be included in the slip models,

147 as mentioned above. To estimate the importance of uncertainly in the slip models and

148 Curie point depths, we calculate the piezomagnetic fields that correspond to the two slip

149 models (USGS and UCSB) with two values of $H(15$ and $30 \mathrm{~km})$. The values for $H$ used

150 here are the same as those used by Utada et al. (2011), and they provide reasonable

151 estimates for the island arc of the Tohoku district and for the subduction zone east of the

152 Tohoku district of Japan (Tanaka et al., 1999). The effects of heterogeneities in the

153 initial magnetization will be discussed separately, later.

\section{Features of the new piezomagnetic models}

piezomagnetic field have been constructed. The spatial distribution of the expected 
enumerate the features that are commonly observed in the results and which correspond

to all sets of parameters. It should be noted that the absolute values given in the results are strongly dependent on the assumed sets of parameters. For this reason, this analysis focuses on relative rather than absolute values.

Relatively large signals of the piezomagnetic field are expected to occur in offshore areas in all cases. For the UCSB slip model, piezomagnetic fields larger than $3 \mathrm{nT}$ are predicted in offshore areas. For the USGS slip models, the predicted piezomagnetic fields are smaller than those for the UCSB model, yet changes larger than $1 \mathrm{nT}$ are predicted. onshore, including the sites of observation, are rather small. Over a vast part of the land area, the predicted amplitudes of the piezomagnetic field are up to $0.4 \mathrm{nT}$. Precise values of the expected changes at the observation sites are listed in Table 2, together with the observed changes reported by Utada et al. (2011). In some models, the changes predicted at some locations are as large as $0.6 \mathrm{nT}$. For example, the predicted change at the ESA site is $0.6 \mathrm{nT}$ for model $\mathrm{b}$ (i.e. UCSB slip model with $H=30 \mathrm{~km}$ ) whereas the predicted change at the same site is zero for other models. There is no location where all the models predict changes greater than $0.4 \mathrm{nT}$. 

that correspond to uniform slip models where the slip parameters are averaged over the fault plane. Numerous differences can be observed between Figs. 1 and 2. For example,

183 the amplitudes of the signals predicted with the uniform slip model do not exceed $1.0 \mathrm{nT}$, except for some localized areas. If we focus on this result, the impression is that the

185 detection of coseismic piezomagnetic signals is a hopeless task, even if the observational area is extended to the seafloor. However, the amplitudes of signals

187 predicted by the heterogeneous slip model are larger than 1-2 nT across a wide area of ocean. In this case, the amplitudes of the coseismic piezomagnetic signals would have been detected, if suitable magnetometers had been installed in the region. highlight the importance of considering the heterogeneous model for the Tohoku-oki earthquake. The large differences also indicate that many of the conclusions about piezomagnetic signals by Utada et al. (2011) need to be reconsidered and probably changed. 


\section{Discussion}

197

The goals of this study were to provide constraints on stress sensitivity, and to

former can be accomplished by comparing the data with the models. The latter can be

202 below.

\subsection{Possible values of the piezomagnetic stress sensitivity} total magnetization $\left(M=\left(M_{x}^{2}+M_{y}^{2}+M_{z}^{2}\right)^{1 / 2}\right)$ are assumed to be given as in Table 1.

211 value of $F_{\mathrm{p}}$ (denoted by $F_{\mathrm{p}}^{\text {calculated }}$ ) is proportional to the assumed value of $\beta M$,

213 yields the disparity between the observed value of $F_{\mathrm{p}}\left(F_{\mathrm{p}}{ }^{\text {observed }}\right)$ and the calculated $F_{\mathrm{p}}$ 
$214\left(F_{\mathrm{p}}^{\text {calculated }}\right)$. The value of $(\beta M)^{\text {actual }}$ is given by

215

$216 \quad(\beta M)^{\text {actual }}=\frac{F_{\mathrm{p}}^{\text {observed }}}{F_{\mathrm{p}}^{\text {calculated }}} \times(\beta M)^{\text {assumed }}$.

218 If $F_{\mathrm{p}}{ }^{\text {observed }}$ and $F_{\mathrm{p}}{ }^{\text {calculated }}$ correlate well, it is possible to determine a plausible value of $219(\beta M)^{\text {actual }}$.

220 Regrettably, the correlation between observed and calculated signals of the

221 piezomagnetic field is not good (Fig. 3). This means that assumptions of a uniform

222 Curie point depth, a uniform initial total magnetization, and/or an assumed slip model,

223 are inadequate. In particular, ignoring the heterogeneity of the initial magnetization is

224 possibly problematic because it is known to enhance the piezomagnetic field (e.g.

225 Oshiman, 1990). Aeromagnetic surveys over the Tohoku region have shown that

226 magnetic anomalies in this region are rather strong (i.e. 10-100 nT) (Fig. 4), raising the

227 possibility of a strong heterogeneity in the initial total magnetization. Consideration of

228 the heterogeneity of the initial magnetization is clearly important if we are to calculate

229 the piezomagnetic field accurately. However, an accurate determination of the structure

230 of the initial magnetization is generally laborious and full of possible errors; hence, an

231 accurate determination of a generated piezomagnetic field is difficult in the presence of 
a strong heterogeneity in the initial magnetization (e.g. Yamazaki, 2011).

Nevertheless, we can attempt to provide constraints on the possible values of the stress sensitivity using data just from sites KAK and KTR. Around these sites, the gradient of the magnetic anomaly is relatively small (Fig. 4), and we can therefore anticipate that the model with uniform initial magnetization will provide reasonable calculated results. The amplitude of the piezomagnetic signal observed at KTR was -0.8 $\pm 0.2 \mathrm{nT}$, whereas those predicted in the theoretical models (Fig. 1a-d) are between -0.2 and $-0.3 \mathrm{nT}$. The piezomagnetic signal observed at KAK was -0.22 , whereas those predicted by theoretical models are between -0.07 and -0.22 . To explain the observations at KAK and KTR, the actual value of $\beta M$ needs to be larger than the value assumed in the present calculation (i.e. $1.0 \mathrm{~Pa}^{-1} \mathrm{Am}^{-1}$ ) by factors of 2-3. Provided that the assumption of $M=1.0 \mathrm{~A} / \mathrm{m}$ (Table 1) is correct, the above result means that the stress sensitivity is about $2.0-3.0 \times 10^{-9} \mathrm{~Pa}^{-1}$. This value is on the same order as that assumed in many piezomagnetic models (e.g. Johnston et al., 1989).

\subsection{Potential usefulness of seafloor magnetic observations}

In all the models of the piezomagnetic field shown in Fig. 2, the amplitudes of the 
250 piezomagnetic signals are expected to be small on land and relatively large offshore.

251 Because the actual value of $\beta M$ is possibly larger than assumed, as discussed in the

252 previous subsection, the actual changes in the magnetic field are possibly larger than

253 those shown in Fig. 1. In particular, large offsets in the magnetic field are expected near

254 the trench axis. Although this result is obtained for an assumption that observations are

255 made at sea level, piezomagnetic signals are also expected to be large at seafloor

256 because the seafloor is rather closer to the rupture. If offshore ocean-bottom

257 magnetometers had been installed, they would have detected significant amplitudes of

258 piezomagnetic signals corresponding to the Tohoku-oki earthquake.

259 Observing piezomagnetic signals would not be very useful if the piezomagnetic

260 signals were insensitive to details of the fault parameters, but the results of the

261 piezomagnetic models demonstrate that this is not the case. Indeed, the spatial

262 distributions of the piezomagnetic field are strongly dependent on the slip model that is

263 adopted. It is possible that we could have improved the determination of earthquake

source parameters if data from ocean-bottom magnetometers had been available, instead of relying solely on the results of inversions of seismic and geodetic data. 
268 are made mainly on land. In the case of the Tohoku-oki earthquake, extremely large

269 slips near the trench axis have been suggested by inversions of the seismic data (e.g.

270 Hayes, 2011; Shao et al., 2011), but better constraints on slip distributions could have

271 been obtained from seafloor geodetic measurements (Sato et al., 2011). Given that

272 seafloor geodetic equipment is costly and difficult to manage, geomagnetic observations

273 might provide useful additional data for monitoring interplate earthquakes along

274 subduction zones. This solution may still apply, even when we consider magnetic

275 anomalies on the seafloor, because heterogeneities in the magnetization of the crust may

276 possibly enhance the generated piezomagnetic field (e.g. Oshiman, 1990).

277 Regrettably, there are also drawbacks to making seafloor magnetic observations.

278 First, seafloor observations are quite costly. Second, it may be difficult to keep the sensors stably located during quakes, and if a sensor is displaced during a quake, an apparent change in the magnetic field will be recorded. Even if it were possible to obtain accurate data of the geomagnetic field at a certain point, it would be necessary to consider heterogeneities of the initial magnetization and ocean-bottom topography when converting the observed changes in the magnetic field to fault source parameters. For these reasons, the usefulness of observing the piezomagnetic field remains uncertain. 
286 observations. Not only are they are extremely costly, but monument stability during

287 earthquakes is also a problem. It is also difficult to process the observations correctly

288 and obtain precise geodetic information. A decision on prioritizing geodetic and

289 geomagnetic techniques should be based on which drawbacks are most easily overcome.

290 If costs allow, an integrated use of both techniques is most desirable because they

291 independently bring useful information to bear on these tectonic phenomena.

292

293

\section{Conclusions}

To calculate the piezomagnetic field that corresponds to the $2011 \mathrm{Mw} 9.0$

Tohoku-oki earthquake, it is necessary to consider the best representation of the spatial

distribution of slip along the fault, and in this paper, I demonstrate the importance of

cannot entirely explain the observed distribution of piezomagnetic signals, constraints 
geomagnetic total forces, due to the piezomagnetic effect, will be relatively large in

offshore areas closer to the rupture, and relatively small onshore, far from the rupture.

Because the expected magnitudes of the piezomagnetic signals are small at existing sites,

this is not tightly constrained. Nevertheless, the possibility of a large piezomagnetic

310 distribution of the piezomagnetic field in oceanic areas are highly dependent on the slip model used. The implication is that detection of the piezomagnetic field with ocean-bottom magnetometers might have provided constraints on the slip models of the

\section{Acknowledgments}

Slip models proposed by Shao et al. (2011) and Hayes (2011), and a program coded

by M. Utsugi, were used to calculate the piezomagnetic fields. Numerical data on the geomagnetic anomalies around Japan were provided by the Geospatial Information 
322 manuscript. The software Generic Mapping Tools (Wessel and Smith, 1998) was used to

323 prepare some of the figures, including maps.

324 


\section{References}

326

Currenti, G., Del Negro, C., Nunnari, G., 2005. Inverse modelling of volcanomagnetic

$$
\text { 10.1111/j.1365-246X.2005.02730.x }
$$

Hayes, G.P., 2011. Rapid source characterization of the 2011 Mw 9.0 off the Pacific coast of Tohoku Earthquake. Earth Planets Space 63, 529-534.

Johnston, M. J. S., 1989. Review of magnetic and electric field effects near active faults and volcanoes in the U.S.A. Phys. Earth Planet. Interiors, 57, 47-63.

Koketsu, K., Yokota, Y., Nishimura, N., Yagi, Y., Miyazaki, S., Satake, K., Fujii, Y., Miyake, H., Yamanaka, Y., Sakai, S., Okada, T., 2011. A unified source model for the 2011 Tohoku earthquake. Earth Planet. Sci. Lett. 310, 480-487. magnetocrystalline anisotropy of magnetite. Phys. Earth Planet. Int. 1, 44-48. by the differential geometric theory of the physical interaction field. Phys. Chem. 
study in the eastern part of Hokkaido, NE Japan: discrepancy between observed and calculated results. Earth Planets Space 56, 1049-1058.

Nishida, Y., Utsugi, M., Mogi, T., 2007. Tectonomagnetic study in the eastern part of Hokkaido, NE Japan (II): Magnetic fields related with the 2003 Tokachi-oki earthquake and the 2004 Kushiro-oki earthquake. Earth Planets Space 59, $1181-1186$.

Okubo, K., Takeuchi, N., Utsugi, M., Yumoto, K., Sasai, Y., 2011. Direct magnetic signals from earthquake rupturing: Iwate-Miyagi earthquake of M 7.2, Japan. Earth Planet. Sci. Lett. 305, 65-72.

Oshiman, N., 1990. Enhancement of tectonomagnetic change due to non-uniform magnetization in the Earth's crust — two dimensional case studies. J. Geomag. Geoelectr. 42, 607-619. observation of piezomagnetic changes due to ground loading by Lake Nichinan, Tottori, Japan. Proceedings of Conductivity Anomaly Symposium (1991), 137-148. (In Japanese) 
361 Sasai, Y., 1994. Piezomagnetic fields produced by dislocation sources. Surv. Geophys.

Sato, M., Ishikawa, T., Ujihara, N., Yoshida, S., Fujita, M., Mochizuki, M., Asada, A., 2011. Displacement Above the Hypocenter of the 2011 Tohoku-Oki Earthquake. Science 332, 1395.

Shao, G., Li, X., Ji. C., Maeda, T., 2011. Focal mechanism and slip history of $2011 \mathrm{Mw}$ 9.1 off the Pacific coast of Tohoku earthquake, constrained with teleseismic body and surface waves. Earth Planets Space 63, 559-564.

Stacey, F.D., Johnston, M.J.S., 1972. The theory of the piezomagnetic effect. Pure Appl. Geophys., 97, 146-155.

Tanaka, A., Okubo, Y., Matsubayashi, O., 1999. Curie point depth based on spectrum analysis of the magnetic anomaly data in east and southeast Asia. Tectonophys. 306, $461-470$.

Utada, H., Shimizu, H., Ogawa, T., Maeda, T., Furumura, T., Yamamoto, T., Yamazaki, N., Yoshitake, Y., Nagamachi, S., 2011. Geomagnetic field changes in response to the 2011 off the Pacific Coast of Tohoku Earthquake and Tsunami. Earth Planet. Sci. Lett. 311, 11-27. 

rectangular fault in a semi-infinite medium. Geophys. J. Int. 140, 479-492.

380 Wessel, P., Smith, W.H.F., 1998. New, improved version of Generic Mapping Tools released. EOS Trans. Am. Geophys. 79, 579. regional stress in inhomogeneously magnetized crust (II): Limitation in general cases. Earth Planets Space 63, 1217-1220. 
Fig. 1. Models of the piezomagnetic field corresponding to the UCSB and USGS slip models. (a) UCSB slip model with $H=15 \mathrm{~km}$. (b) UCSB slip model with $H=30$ km. (c) USGS slip model with $H=15 \mathrm{~km}$. (d) USGS slip model with $H=30 \mathrm{~km}$.

Fig. 2. Models of the piezomagnetic field corresponding to averaged versions of the slip is considered. four models. Error bars in observed values are from Utada et al. (2011). Open circles indicate the results at sites KAK and KTR, where the magnetic anomalies 

are rather small, while solid circles indicate the results at other sites.

Fig. 4. The magnetic anomaly over the Tohoku region as observed by an aeromagnetic survey at a height of $5000 \mathrm{~m}$. Contour intervals are $10 \mathrm{nT}$. Observations were conducted by the Geographical Survey Institute (predecessor of the Geospatial Information Authority) of Japan in 1990, and the data are available on their Web site, in Japanese (http://vldb.gsi.go.jp/sokuchi/geomag/menu_03/aeromag_data.html; last access: 26 October 2012).

415

416 
(a)

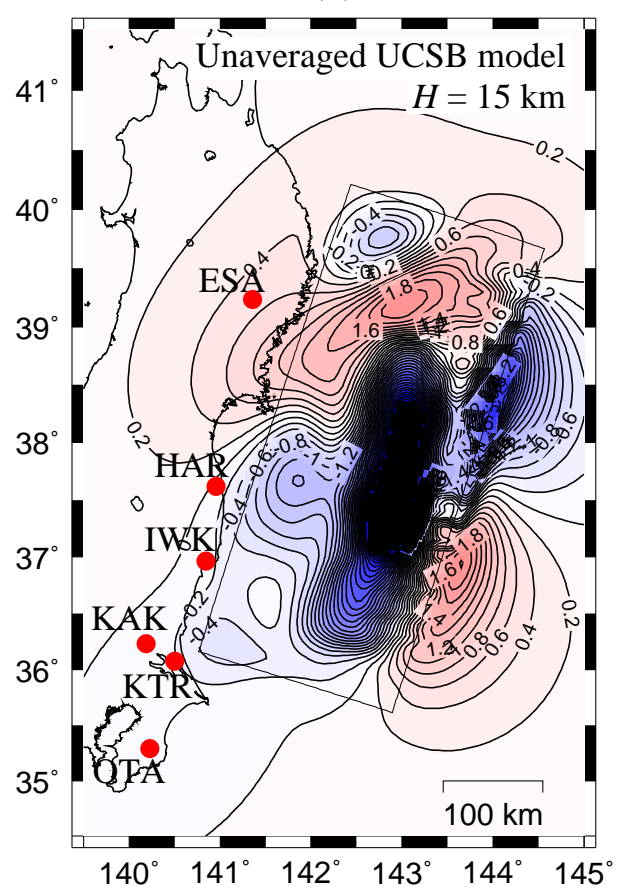

(c)

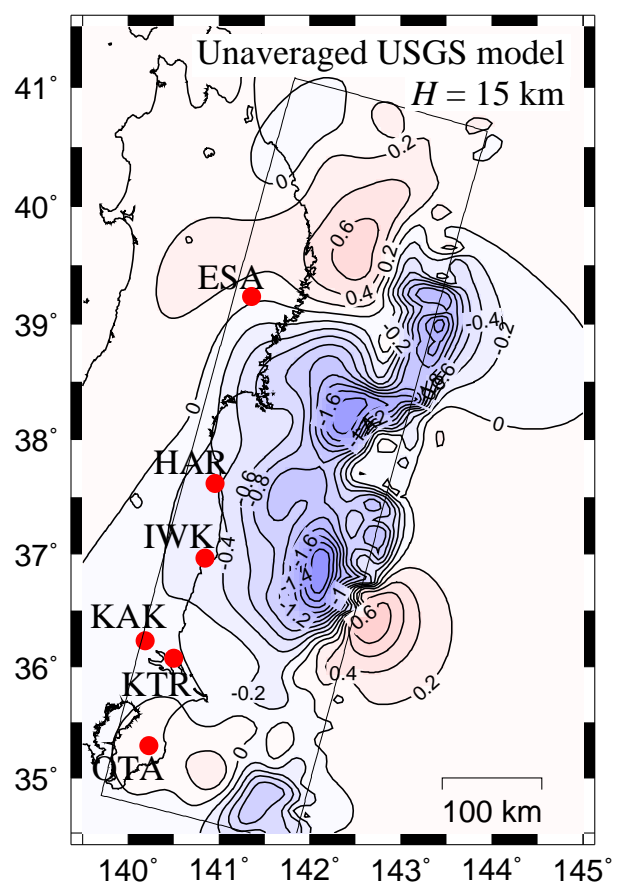

(b)

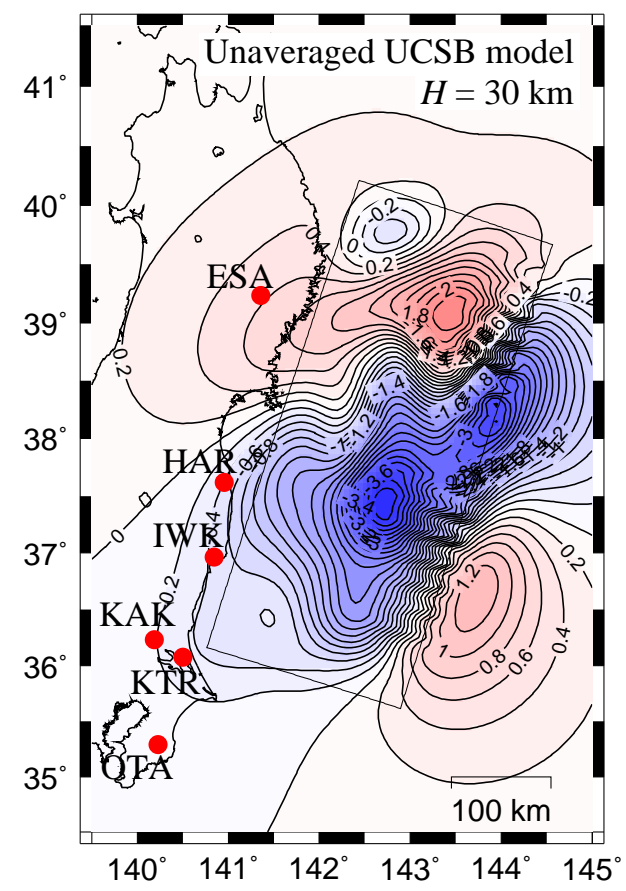

(d)

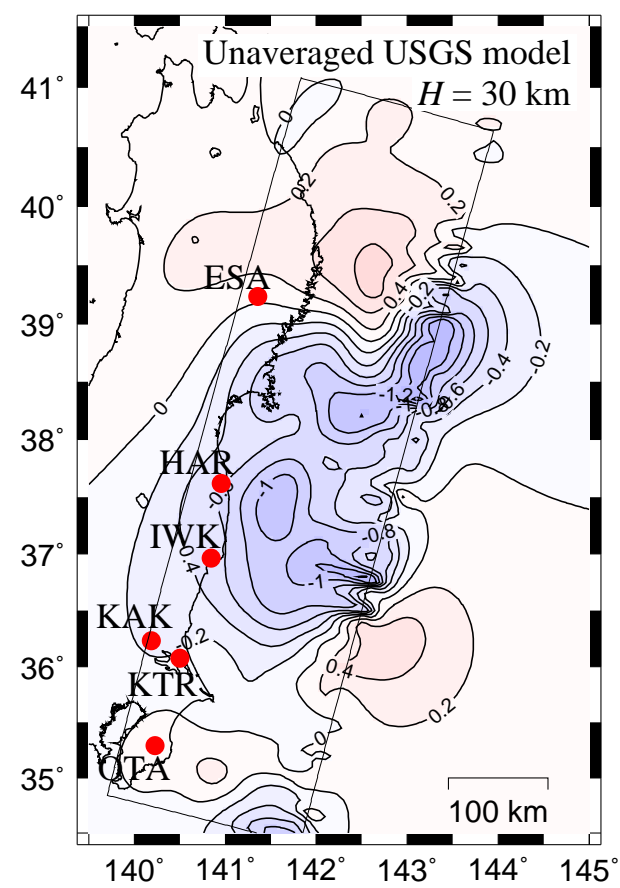

Figure 1 Models of the piezomagnetic field corresponding to the UCSB and USGS slip models. (a) UCSB slip model with $H=15 \mathrm{~km}$. (b) UCSB slip model with $H=30 \mathrm{~km}$. (c) USGS slip model with $H=15 \mathrm{~km}$. (d) USGS slip model with $H=30 \mathrm{~km}$. The rectangle represents the fault plane on which heterogeneous slip is considered. 
(a)

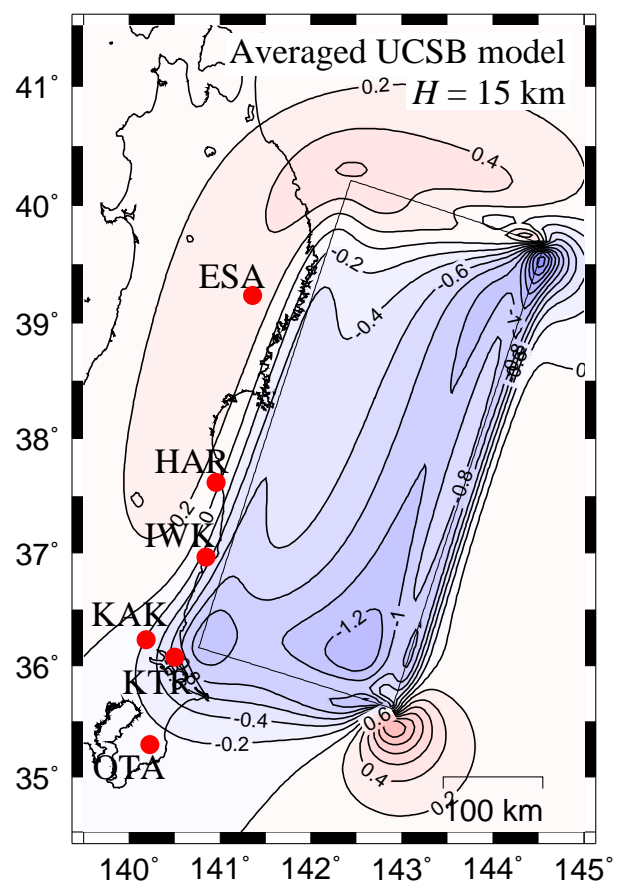

(c)

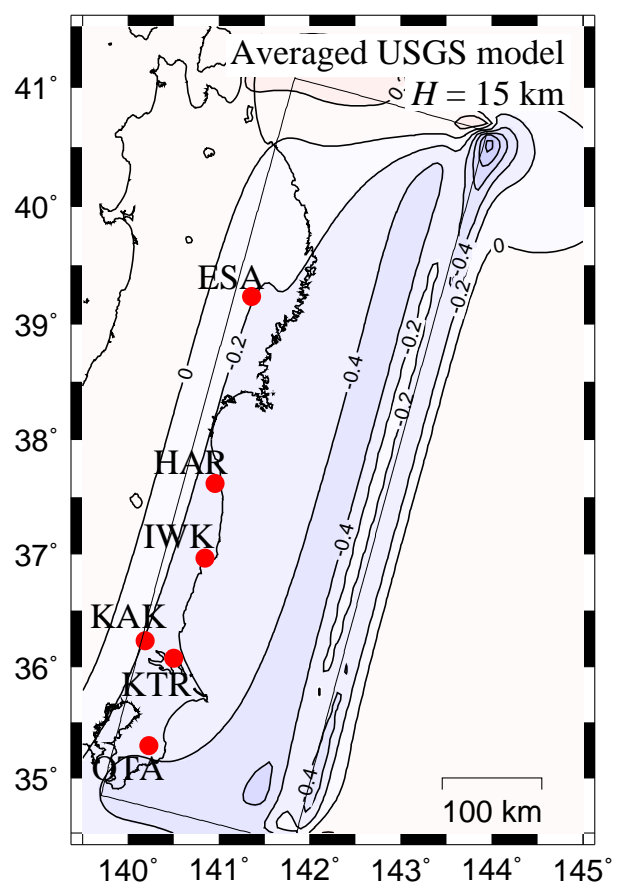

(b)

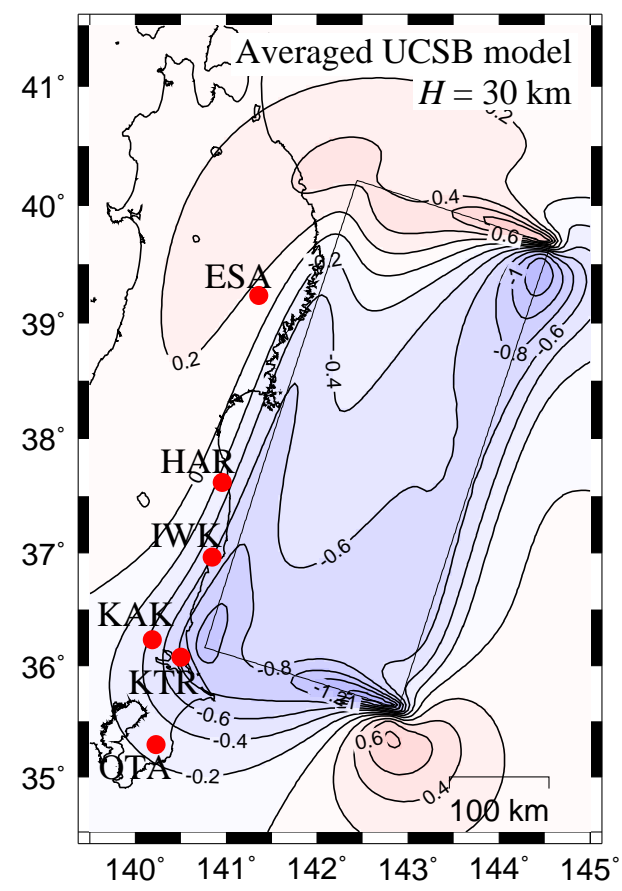

(d)

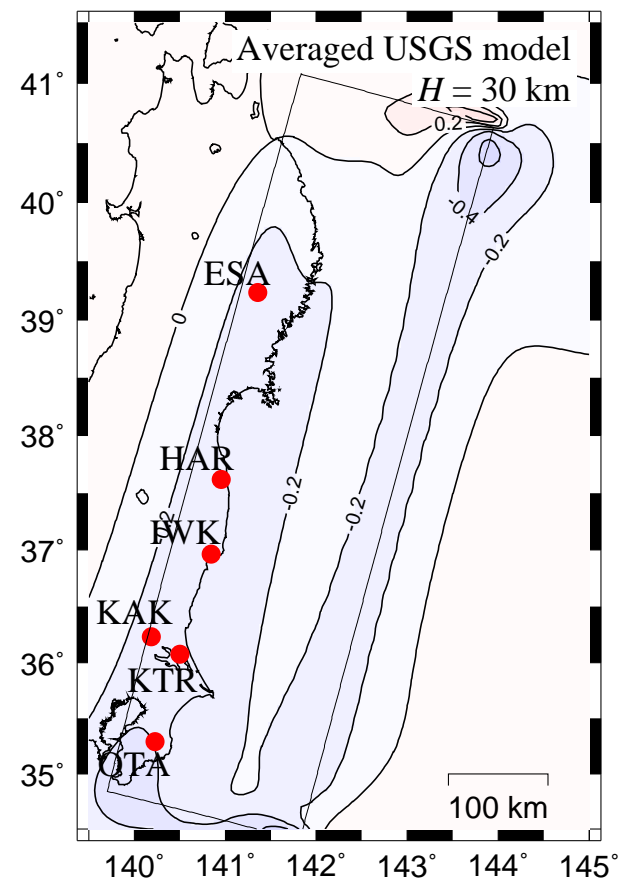

Figure 2 Models of the piezomagnetic field corresponding to averaged versions of the UCSB and USGS slip models. (a) UCSB slip model with $H=15 \mathrm{~km}$. (b) UCSB slip model with $H=30 \mathrm{~km}$. (c) USGS slip model with $H=15 \mathrm{~km}$. (d) USGS slip model with $H=30 \mathrm{~km}$. The rectangle represents the fault plane on which uniform slip is considered. 


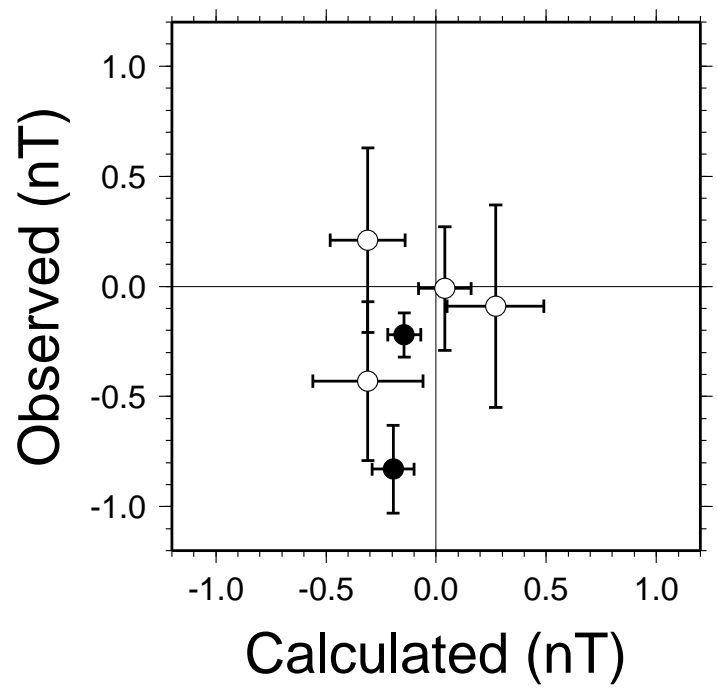

Figure 3 Comparisons between observed and calculated piezomagnetic signals. Calculated values in this figure are the averages of four piezomagnetic models. Error bars in calculated values represent maximum and minimum values for the four models. Error bars in observed values are from Utada et al. (2011). Solid circles indicate the results at sites KAK and KTR, where the magnetic anomalies are rather small, while open circles indicate the results at other sites. 


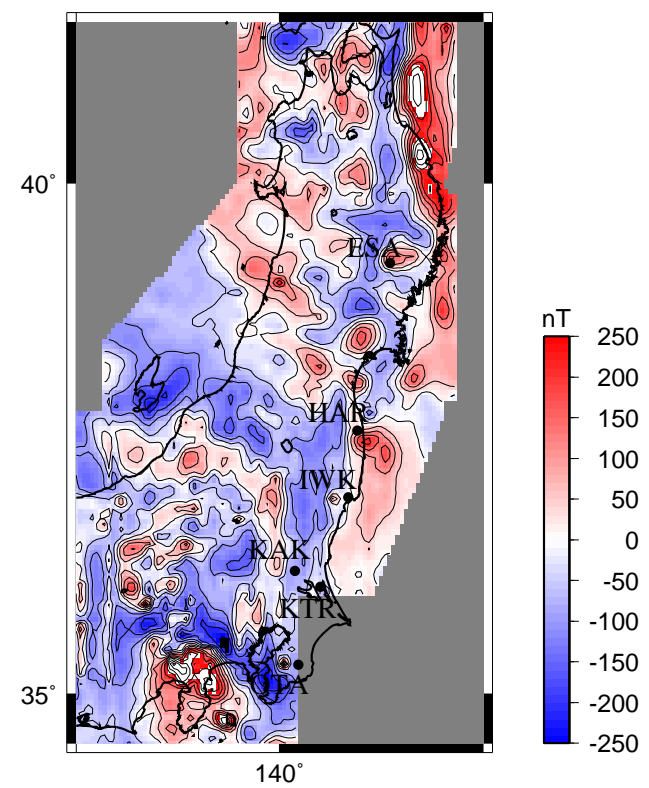

Figure 4 The magnetic anomaly over the Tohoku region as observed by an aeromagnetic survey at a height of $5000 \mathrm{~m}$. Contour intervals are $10 \mathrm{nT}$. Observations were conducted by the Geographical Survey Institute (predecessor of the Geospatial Information Authority) of Japan in 1990, and the data are available on their Web site, in Japanese (http://vldb.gsi.go.jp/sokuchi/geomag/menu_03/aeromag_data.html; last access: 26 October 2012). 


\section{Table 1}

Parameters assumed in the modeling of the piezomagnetic field.

\begin{tabular}{ll} 
Parameter & Value \\
\hline Rigidity & $57(53) \times 10^{9} \mathrm{~Pa}$ \\
Poisson's ratio & 0.25 \\
Magnetization & $1.0 \mathrm{~A} \mathrm{~m}^{-1}$ \\
Piezomagnetic & $1 \times 10^{-9} \mathrm{~Pa}^{-1}$ \\
stress sensitivity & 15 and $30 \mathrm{~km}$ \\
Curie point depth & 51.0 degree \\
Inclination of the ambient & \\
geomagnetic field & -7.5 degree \\
Declination of the ambient & \\
geomagnetic field & $0 \mathrm{~m}$ \\
Observation altitude &
\end{tabular}


Table 2

Comparisons of coseismic changes in the geomagnetic total intensity, as predicted by the piezomagnetic models versus those calculated from data reported in Utada et al. (2011). Piezomagnetic field models are determined for Curie point depths of 30 and 15 km together with two slip models (USGS and UCSB).

\begin{tabular}{lccccccc} 
& \multicolumn{2}{c}{ Station code } & \multicolumn{2}{c}{ USGS slip model } & \multicolumn{2}{c}{ UCSB slip model } & \multicolumn{2}{c}{ Observed (error) } \\
& $30 \mathrm{~km}$ & $15 \mathrm{~km}$ & $30 \mathrm{~km}$ & $15 \mathrm{~km}$ & & \\
\hline ESA & +0.05 & +0.05 & +0.55 & +0.49 & -0.09 & $(0.46)$ \\
HAR & -0.56 & -0.37 & -0.40 & -0.06 & -0.43 & $(0.36)$ \\
IWK & -0.48 & -0.28 & -0.44 & -0.14 & +0.21 & $(0.42)$ \\
KTR & -0.18 & -0.10 & -0.29 & -0.18 & -0.83 & $(0.20)$ \\
OTA & +0.14 & +0.16 & -0.08 & -0.03 & -0.01 & $(0.28)$ \\
KAK & -0.22 & -0.14 & -0.19 & -0.07 & -0.22 & $(0.10)$ \\
\hline
\end{tabular}

